\title{
A Kind of Stochastic Eigenvalue Complementarity Problems
}

\author{
Ying-xiao Wang and Shou-qiang Du \\ School of Mathematics and Statistics, Qingdao University, Qingdao 266071, China \\ Correspondence should be addressed to Shou-qiang Du; sqdu@qdu.edu.cn
}

Received 23 October 2017; Revised 22 December 2017; Accepted 23 January 2018; Published 20 February 2018

Academic Editor: Sergey Tkachenko

Copyright (C) 2018 Ying-xiao Wang and Shou-qiang Du. This is an open access article distributed under the Creative Commons Attribution License, which permits unrestricted use, distribution, and reproduction in any medium, provided the original work is properly cited.

With the development of computer science, computational electromagnetics have also been widely used. Electromagnetic phenomena are closely related to eigenvalue problems. On the other hand, in order to solve the uncertainty of input data, the stochastic eigenvalue complementarity problem, which is a general formulation for the eigenvalue complementarity problem, has aroused interest in research. So, in this paper, we propose a new kind of stochastic eigenvalue complementarity problem. We reformulate the given stochastic eigenvalue complementarity problem as a system of nonsmooth equations with nonnegative constraints. Then, a projected smoothing Newton method is presented to solve it. The global and local convergence properties of the given method for solving the proposed stochastic eigenvalue complementarity problem are also given. Finally, the related numerical results show that the proposed method is efficient.

\section{Introduction}

Computational electromagnetics is a science, which spans many subjects. It is an organic combination of mathematical theory, electromagnetic theory, and computer science. Electromagnetism is the classical dynamics theory. Electromagnetic phenomena are also closely related to eigenvalue problems and essentially can be deduced by Maxwell eigenvalue equation. The typical eigenvalue problem of the electromagnetic field is the resonant problem of the cavity and the guide wave problem of the waveguide. No matter the resonance problem of closed cavity, or the propagation problem of uniform guided waves, the wave equation is homogeneous without considering any source or field excitation process. For simplification, the conductors that constitute the cavity are idealized. So the field satisfies the homogeneous boundary condition on the boundary. There are many methods for solving computational electromagnetics, such as finite difference method and finite element method; see literatures [1-7] for details. In recent years, various methods in computational electromagnetics have been continuously improved. However, the input data are usually affected by many uncertain factors, such as the environment, which often results in the difference between calculation and measurement. So, we consider establishing a new model to solve some related electromagnetics problems. This is also the motivation of this paper.

Among the optimization model, as we all know, the eigenvalue complementarity problem arises from several important applications in engineering and physics, such as the study of the resonance frequency and the stability of dynamic systems. In the last few years, the eigenvalue complementarity problem has drawn increasing attention, in many literature systems, such as [8-13] and the references therein. Among them, in [8], the authors study an eigenvalue complementarity problem and find its origins in the solution of a contract problem in mechanics. In [9], the eigenvalue complementarity problems with symmetric real matrices are considered. The authors transform this problem into a differentiable optimization program involving the Rayleigh quotient on a simplex and find its stationary point by the spectral projected gradient algorithm. In [10-13], many methods are proposed to solve the eigenvalue complementarity problems, such as Levenberg-Marquardt method and the derivative-free projection method. In [14], the stability of dynamic system is studied. The model of eigenvalue complementarity problem is established and the finite element method is used to solve the model. 
On the other hand, the stochastic linear complementarity problems, stochastic nonlinear complementarity problems, and some related stochastic optimization problems attract many researchers' attention. And a lot of related theories and algorithms are given, such as [15-20] and their references. The expected value (EV) to reformulate the problems is usually used. Many methods are effectual for solving the stochastic complementarity problems. The global and local convergence of these methods are also given. Besides, the expected residual minimization (ERM) also can reformulate the stochastic complementarity problems, such as those in [21-23]. And the stochastic complementarity problems are eventually transformed into a certain minimal problem with constraints.

In order to reduce the difference between calculation and measurement in computational electromagnetics, we consider getting the approximate distribution, by statistics, of a large number of experimental data and establishing a new stochastic model. So, in this paper, we propose a new kind of stochastic eigenvalue complementarity problem and the smoothing Newton method is also proposed to solve the given problem.

Denote $(\Omega, F, P)$ as a probability space with $\Omega \subseteq R^{n}$, where the probability distribution $P$ is known. The new kind of stochastic eigenvalue complementarity problem is to find a scalar $\lambda>0$ and a vector $x \in R^{n} \backslash\{0\}$, such that

$$
u=(\lambda B(\omega)-A(\omega)) x, \quad x \geq 0, u \geq 0, x^{T} u=0,
$$

where $A(\omega)$ and $B(\omega)$ are $n \times n$ symmetric positive definite stochastic matrices, $\Omega \subseteq R^{n}$ is the probability space, and $\omega \in$ $\Omega=\left\{\omega_{1}, \omega_{2}, \ldots, \omega_{m}\right\}$ is a random vector given in probability distribution $P$.

The rest of this paper is organized as follows. In Section 2, we reformulate problem (1) as a nonsmooth system firstly and give the related preliminary results. In Section 3, we present the projected smoothing Newton method and give the convergence results. In Section 4, some preliminary numerical results are proposed. The last section is the conclusion.

\section{Preliminary Results}

In this section, we give the reformulation of problem (1) and some related preliminaries.

Firstly, we consider using the expected value model to solve (1) as the following equations.

Denote

$$
\begin{aligned}
& \bar{B}=\sum_{i=1}^{m} p_{i} B\left(\omega_{i}\right), \\
& \bar{A}=\sum_{i=1}^{m} p_{i} A\left(\omega_{i}\right),
\end{aligned}
$$

where $p_{i}=P\left(\omega_{i} \in \Omega\right) \geq 0, i=1, \ldots, m$. So (1) is equivalent to the problem, which is defined as

$$
x \geq 0
$$

$$
\begin{aligned}
(\lambda \bar{B}-\bar{A}) x & \geq 0, \\
x^{T}(\lambda \bar{B}-\bar{A}) x & =0, \\
\left(\lambda B\left(\omega_{i}\right)-A\left(\omega_{i}\right)\right) x & \geq 0, \quad i=1, \ldots, m .
\end{aligned}
$$

As (3) is a linear complementarity problem, we can use the complementarity functions to transform it. The complementarity function has many different forms, such as the functions given in [24-29]. Among them, the Fischer-Burmeister (FB) function

$$
\phi(x)=\sqrt{a^{2}+b^{2}}-(a+b)
$$

is used widely. By the FB function transformation, (3) is equivalent to $\Phi(x)=0$, where

$$
\Phi(x)=\left(\begin{array}{c}
\phi\left(x_{1},(\lambda \bar{B}-\bar{A})_{1} x\right) \\
\vdots \\
\phi\left(x_{n},(\lambda \bar{B}-\bar{A})_{n} x\right)
\end{array}\right) \text {. }
$$

In this paper, we consider using the smoothing FischerBurmeister function, which is differentiable at any point. This smoothing method, which is an important method to solve nonsmooth problems, has been widely used in recent years, such as in [30-33]. The idea of a smoothing method is to approximate nonsmooth functions by a sequence of smooth functions. There are many forms of smooth functions. Qi and Chen proposed a smooth approximation method for nonsmooth functions in [34]. By introducing the smoothing approximation function of $\Phi_{\mu}: R^{n} \rightarrow R^{n}, \mu>0$, smoothing methods are proposed by Chen et al. So far, a lot of progress has been made in the study of smoothing methods. In this paper, similar to [28], we use the smoothing FischerBurmeister function as

$$
\phi_{\mu}(a, b)=\sqrt{a^{2}+b^{2}+\mu}-(a+b),
$$

where $\mu>0$.

Therefore, (3) is equivalent to $\Phi_{\mu}(x)=0$, where

$$
\Phi_{\mu}(x)=\left(\begin{array}{c}
\phi\left(x_{1},(\lambda \bar{B}-\bar{A})_{1} x\right) \\
\vdots \\
\phi\left(x_{n},(\lambda \bar{B}-\bar{A})_{n} x\right)
\end{array}\right) .
$$

Then, (3) and (4) are equivalent to the following problem:

$$
H(x, \lambda, \mu, y)=0, \quad y \geq 0, \lambda>0, \mu>0,
$$


where

$$
\begin{aligned}
H(x, \lambda, \mu, y) & =\left(\begin{array}{c}
\Phi_{\mu}(x) \\
\left(\lambda B\left(\omega_{1}\right)-A\left(\omega_{1}\right)\right) x-y_{1} \\
\vdots \\
\left(\lambda B\left(\omega_{m}\right)-A\left(\omega_{m}\right)\right) x-y_{m} \\
x^{T} x-1 \\
2^{\mu}-1
\end{array}\right) \\
& =0
\end{aligned}
$$

and $y=\left(y_{1}^{T}, \ldots, y_{m}^{T}\right)^{T} \in R^{m n}$. Let $z=\left(x^{T}, \lambda, \mu, y^{T}\right)^{T} \epsilon$ $R^{(m+1) n+2}$ and define a merit function of (9) as $f(z)=$ $(1 / 2)\|H(z)\|^{2}$.

If (1) has a solution, then solving (9) is equivalent to finding a global solution of the following optimization problem:

$$
\begin{aligned}
\min & f(z) \\
\text { s.t. } & z \in Z,
\end{aligned}
$$

where $Z=\left\{\left(x^{T}, \lambda, \mu, y^{T}\right)^{T} \mid x \geq 0, \lambda>0, \mu>0, y \geq 0\right\}$.

Next, we give some related definitions and propositions.

Definition 1. Assume that $F: R^{m} \rightarrow R^{n}$ is a locally Lipschitzian function. The $B$-subdifferential of $F$ at $x$ is

$$
\begin{gathered}
\partial_{B} F(x)=\left\{V \in R^{m \times n} \mid \exists\left\{x_{k}\right\} \subseteq D_{F}:\left\{x_{k}\right\}\right. \\
\left.\longrightarrow x, F^{\prime}\left(x_{k}\right) \longrightarrow V\right\},
\end{gathered}
$$

where $D_{F}$ is the differentiable points set and $F^{\prime}\left(x_{k}\right)$ is the Jacobian of $F$ at a point $x \in R^{n}$.

Here, for any $z=\left(x^{T}, \lambda, \mu, y^{T}\right)^{T} \in R^{(m+1) n+2}$, we have

$$
\begin{aligned}
& \partial_{B} H(z) \\
& =\left(\begin{array}{cccccc}
V_{\Phi_{\mu}} & V_{\Phi_{\mu}}^{\prime} & V_{\Phi_{\mu}}^{\prime \prime} & 0 & \cdots & 0 \\
\lambda B\left(\omega_{1}\right)-A\left(\omega_{1}\right) & B\left(\omega_{1}\right) & 0 & -I & \cdots & 0 \\
\vdots & \vdots & \vdots & \vdots & \vdots & \vdots \\
\lambda B\left(\omega_{m}\right)-A\left(\omega_{m}\right) & B\left(\omega_{m}\right) & 0 & 0 & \cdots & -I \\
2 x & 0 & 0 & 0 & 0 & 0 \\
0 & 0 & 0 & 2^{\mu} \ln 2 & 0 & 0
\end{array}\right),
\end{aligned}
$$

where $V_{\Phi_{\mu}}$ is the $B$-subdifferential of $\Phi_{\mu}$ to $x, V_{\Phi_{\mu}}^{\prime}$ is the $B$-subdifferential of $\Phi_{\mu}$ to $\lambda$, and $V_{\Phi_{\mu}}^{\prime \prime}$ is the $B$-subdifferential of $\Phi_{\mu}$ to $\mu$. $I$ is the $n \times n$ identity matrix and $\lambda \in R_{+}, \mu \in R_{+}$.

Definition 2 (see [18]). F is said to be semismooth at $x$ if

$$
\lim _{V \in \partial_{B} F\left(x+t h^{\prime}\right), h^{\prime} \rightarrow h, t \downarrow 0} V h^{\prime}
$$

exist for any $h \in R^{n}$.
Definition 3 (see [18]). $F$ is said to be strongly semismooth at $x$ if $F$ is semismooth at $x$ and for any $V \in \partial F(z+h), h \rightarrow 0$,

$$
\lim _{h \rightarrow 0, V \in \partial_{B} F(x+h)} \frac{\|F(x+h)-F(x)-V h\|}{\|d\|^{2}}<\infty .
$$

Definition 4 (see [35]). $F$ is said to be $B D$-regular at a point $x$ if all the elements in $\partial_{B} F(x)$ are nonsingular.

\section{Projected Smoothing Newton Method and Convergence Analysis}

In this section, we present the projected smoothing Newton method and establish the global and local convergence results of the given method.

Method 1 (projected smoothing Newton method).

Step 0 . Choose $\sigma \in(0,1), \gamma \in(0,1), \bar{\mu} \in R_{+}$satisfying $\gamma \bar{\mu}<$ $1, \beta \in(0,1)$.

Step 1. Compute $g_{k}=\nabla f\left(z_{k}\right)$. If $\left\|g_{k}\right\|<\epsilon$, stop.

Step 2. Compute $d_{k}$ by

$$
H\left(z_{k}\right)+\left(V_{k}+\alpha I\right) d_{k}=\beta \bar{z},
$$

where $V_{k} \in \partial_{B} H\left(z_{k}\right), \alpha \geq 0, \bar{z}=(\bar{\mu}, 0) \in R^{((m+1) n+2)}$.

Step 3. Let $m$ be the least nonnegative integer satisfying

$$
f\left(P_{Z}\left(z_{k}+\beta^{m} d_{k}\right)\right) \leq\left(1-2 \sigma(1-\gamma \bar{\mu}) \beta^{m}\right) f\left(z_{k}\right) .
$$

Let $z_{k+1}=P_{Z}\left(z_{k}+\beta^{m} d_{k}\right)$.

Step 4 . Set $k:=k+1$; return to Step 1 .

In the following, we give the convergence results of Method 1.

Theorem 5. Let $\left\{z_{k}\right\}$ be an infinite sequence generated by Method 1 , and $z^{*}$ is an accumulation point of $\left\{z_{k}\right\}$. If $z^{*}$ is a $B D$-regular solution of $f\left(z_{k}\right), z^{*}$ is a solution of $H(z)=0$.

Proof. From Step 3 of Method 1, we have $f\left(z_{k+1}\right)<f\left(z_{k}\right)$, for all $k \geq 0$. Hence, $f\left(z_{k}\right)$ is monotonically decreasing. Since $\left(z_{k}\right) \geq 0(k \geq 0)$, there exists $f^{*}$, such that $\lim _{k \rightarrow \infty} f\left(z_{k}\right)=$ $f^{*}=f\left(z^{*}\right)$.

We suppose $f\left(z^{*}\right)>0$. By (17), we have

$$
f\left(z_{k+1}\right) \leq\left(1-2 \sigma(1-\gamma \bar{\mu}) \beta^{m}\right) f\left(z_{k}\right) .
$$

Take the limit of (18) on both sides. We get

$$
f\left(z^{*}\right) \leq\left(1-2 \sigma(1-\gamma \bar{\mu}) \beta^{m}\right) f\left(z^{*}\right) .
$$

Then, we have $\left(2 \sigma(1-\gamma \bar{\mu}) \beta^{m}\right) f\left(z^{*}\right) \leq 0$.

This is in contradiction to $\sigma \in(0,1), \gamma \bar{\mu}<1$. Hence, $H\left(z^{*}\right)=0$. The proof is completed.

Theorem 6. Let $\left\{z_{k}\right\}$ be an infinite sequence generated by Method 1 and $z^{*}$ be a BD-regular solution; the rate of convergence is quadratic. 
Proof. By Proposition 2.1.1(b) in [36] and $\partial_{B} H\left(z_{k}\right)$ being nonempty compact set at any point, we have that $V_{k} \in$ $\partial_{B} H\left(z_{k}\right)$ is bounded in bounded set of points. So, there is a constant $c_{1}>0$ and $c_{3}>0$, such that, for all $z_{k} \in \bigcup\left(z^{*}\right.$, $\left.\delta_{1}\right)$, where $\delta_{1}$ is a sufficiently small positive constant, $\|\left(V_{k}+\right.$ $\alpha I)^{-1} \| \leq c_{1}$ and $\left\|V_{k}\right\| \leq c_{3}$ hold. Besides, by Definition 3, there exists a positive constant $c_{2}$, such that

$$
\left\|H\left(z_{k}\right)-H\left(z^{*}\right)-V_{k}\left(z_{k}-z^{*}\right)\right\| \leq c_{2}\left\|z_{k}-z^{*}\right\|^{2},
$$

for all $z_{k} \in \bigcup\left(z^{*}, \delta_{1}\right)$, where $\delta_{2}$ is a sufficiently small positive constant. Moreover, there exists a positive constant $c_{4}$, such that $\beta \bar{z}=c_{4}\left\|z_{k}-z^{8}\right\|^{2}$ hold.

Denote $\delta=\min \left(\delta_{1}, \delta_{2}\right)$, for all $z_{k} \in \bigcup\left(z^{*}, \delta\right)$. We have

$$
\begin{aligned}
& \left(V_{k}+\alpha I\right)\left(z_{k+1}-z^{*}\right)=\left(V_{k}+\alpha I\right) \\
& \cdot\left(P_{Z}\left(z_{k}+\beta^{m} d_{k}\right)-z^{*}\right)=\left(V_{k}+\alpha I\right) \\
& \cdot\left(z_{k}+d_{k}-z^{*}+P_{Z}\left(z_{k}+\beta^{m} d_{k}\right)-\left(z_{k}+d_{k}\right)\right) \\
& =\left(V_{k}+\alpha I\right)\left(z_{k}-z^{*}+d_{k}\right)+\left(V_{k}+\alpha I\right) \\
& \cdot\left(P_{Z}\left(z_{k}+\beta^{m} d_{k}\right)-\left(z_{k}+d_{k}\right)\right)=\left(V_{k}+\alpha I\right) \\
& \cdot\left(z_{k}-z^{*}\right)+\left(V_{k}+\alpha I\right) d_{k}+\left(V_{k}+\alpha I\right) \\
& \cdot\left(P_{Z}\left(z_{k}+\beta^{m} d_{k}\right)-\left(z_{k}+d_{k}\right)\right)=V_{k}\left(z_{k}-z^{*}\right) \\
& +\alpha\left(z_{k}-z^{*}\right)+\beta \bar{z}-H\left(z_{k}\right)+\left(V_{k}+\alpha I\right) \\
& \cdot\left(P_{Z}\left(z_{k}+\beta^{m} d_{k}\right)-\left(z_{k}+d_{k}\right)\right) .
\end{aligned}
$$

Since $H$ is a locally Lipschitzian function, we premultiply this equation by $\left(V_{k}+\alpha I\right)^{-1}$ and take norms on both sides. So we get

$$
\begin{aligned}
& \left\|z_{k+1}-z^{*}\right\| \leq\left\|\left(V_{k}+\alpha I\right)^{-1}\right\| \| V_{k}\left(z_{k}-z^{*}\right) \\
& +\alpha\left(z_{k}-z^{*}\right)-H\left(z_{k}\right) \\
& \quad+\left(V_{k}+\alpha I\right)\left(P_{Z}\left(z_{k}+\beta^{m} d_{k}\right)-\left(z_{k}+d_{k}\right)\right)+\beta \bar{z} \| \\
& \quad \leq c_{1}\left(\left\|H\left(z^{*}-H\left(z_{k}\right)+V_{k}\left(z_{k}-z^{*}\right)\right)\right\|\right. \\
& \left.+\alpha\left\|z_{k}-z^{*}\right\|\right)+\left\|P_{Z}\left(z_{k}+\beta^{m} d_{k}\right)-\left(z_{k}+d_{k}\right)\right\| \\
& +\|\beta \bar{z}\| \leq c_{1}\left(c_{2}+c_{3}\right)\left\|z_{k}-z^{*}\right\|^{2}+\left\|z_{k}+d_{k}-z^{*}\right\| \\
& +c_{4}\left\|z_{k}-z^{*}\right\|^{2} \leq c_{1}\left(c_{2}+c-3+c_{4}\right)\left\|z_{k}-z^{*}\right\|^{2} \\
& +\left\|z_{k}-z^{*}+\left(V_{k}+\alpha I\right)^{-1}\left(\beta \bar{z}-H\left(z_{k}\right)\right)\right\| \leq c_{1}\left(c_{2}\right. \\
& \left.+c-3+c_{4}\right)\left\|z_{k}-z^{*}\right\|^{2}+c_{1}\left(c_{4}\left\|z_{k}-z^{*}\right\|^{2}\right. \\
& \left.\quad+c_{2}\left\|z_{k}-z^{*}\right\|^{2}+c_{3}\left\|z_{k}-z^{*}\right\|^{2}\right)=2 c_{1}\left(c_{2}+c_{3}+c_{4}\right) \\
& \quad+\left\|z_{k}-z^{*}\right\|^{2}=\tau\left\|z_{k}-z^{*}\right\|^{2},
\end{aligned}
$$

where $\tau=2 c_{1}\left(c_{2}+c_{3}+c_{4}\right)$. Therefore, similar to the proof of Theorem 2.3 in [37], we know that the rate of convergence is $Q$-quadratic. The proof is completed.

\section{Numerical Results}

In this section, we give some related numerical experiments. Because problem (1) is proposed for the first time, we give the following examples combined with the examples in literatures $[1-7,38]$. In [7], the authors consider the complex shell inside the complicated enclosures of the electronic component and the high frequency radiation. The enclosure is assumed to be large compared to the wavelength. In this case, wavelengths have strong dependence on the properties of waves, including eigenvalues, eigenfunctions, scattering, and impedance matrices under small perturbations. The field fluctuations within the enclosure are described in a statistical sense using random matrix theory. In [38], in the microwave frequency band, the resonant circuit is usually realized by a resonant cavity. The cavity is filled with air or other mediums, and the electromagnetic oscillation in the cavity can be generated in the cavity by the way of probe and small hole. The waveguide cavity is formed by sealing the waveguides along the ends of the propagation direction with a conductor. Example 10 is converted from a two-dimensional rectangular resonant cavity problem in random system, which is proposed in [3, 38]. The program is written in Matlab 7.0. The parameters in Method 1 are taken as

$$
\begin{aligned}
\delta & =0.1, \\
\sigma & =0.1, \\
\mu_{0} & =1, \\
\bar{\mu} & =1, \\
\gamma & =0.1 .
\end{aligned}
$$

The stopping rules for Method 1 are $\left\|\nabla f\left(z_{k}\right)\right\| \leq 1 e-3$ or $k_{\text {max }}=2 \times 10^{4}$.

The initial points are randomly selected. In these tables of the numerical results, $x^{*}$ denotes the point of the minimization of $f(z), \lambda$ denotes the eigenvalue, and DIM denotes the dimension of the problem.

Example 7. We consider problem (1), where

$$
\begin{aligned}
A(\omega) & =\left(\begin{array}{cc}
3+\omega & 1 \\
1 & 2+\omega
\end{array}\right), \\
B(\omega) & =\left(\begin{array}{cc}
2+\omega & 1 \\
1 & 1+\omega
\end{array}\right), \\
\Omega & =\{0,1\}, \\
p_{i} & =P\left(\omega_{i} \in \Omega\right)=0.5, \quad i=1,2 .
\end{aligned}
$$

The numerical results of Example 7 are given in Table 1 and the values of $f(z)$ at each iteration are shown in Figure 1. 
TABLE 1: Numerical results of Example 7.

\begin{tabular}{lcc}
\hline$\lambda$ & $x^{*}$ & $f\left(z^{*}\right)$ \\
\hline 1.5343 & $(0.3447,0.2414)^{T}$ & 0.4328 \\
1.5280 & $(0.3827,0.3481)^{T}$ & 0.4193 \\
1.5159 & $(0.4038,0.3369)^{T}$ & 0.3759 \\
1.5472 & $(0.3267,0.1698)^{T}$ & 0.4327 \\
1.4130 & $(0.6240,0.3500)^{T}$ & 0.1902 \\
\hline
\end{tabular}

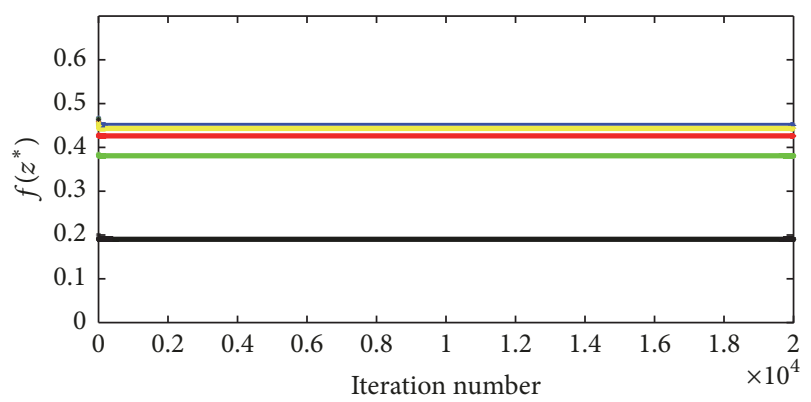

- The 1st numerical experiment

- The 2nd numerical experiment

* The 3rd numerical experiment

The 4th numerical experiment

- The 5 th numerical experiment

FIGURE 1: Change curve of numerical value of $f(z)$ at every iteration.

Example 8. We consider problem (1), where

$A(\omega)$

$$
=\left(\begin{array}{cccccc}
2+\omega & 1 & 1 & 1 & 1 & 1 \\
1 & 2+\omega & 1 & 1 & 1 & 1 \\
1 & 1 & 2+\omega & 1 & 1 & 1 \\
1 & 1 & 1 & 2+\omega & 1 & 1 \\
1 & 1 & 1 & 1 & 2+\omega & 1 \\
1 & 1 & 1 & 1 & 1 & 2+\omega
\end{array}\right),
$$

$B(\omega)$

$$
=\left(\begin{array}{cccccc}
3+\omega & 1 & 1 & 1 & 1 & 1 \\
1 & 3+\omega & 1 & 1 & 1 & 1 \\
1 & 1 & 3+\omega & 1 & 1 & 1 \\
1 & 1 & 1 & 3+\omega & 1 & 1 \\
1 & 1 & 1 & 1 & 3+\omega & 1 \\
1 & 1 & 1 & 1 & 1 & 3+\omega
\end{array}\right),
$$

$\Omega=\{0,1\}$,

$p_{i}=P\left(\omega_{i} \in \Omega\right)=0.5, \quad i=1,2$.

The numerical results of Example 8 are shown in Table 2 and the values of $f(z)$ at each iteration are shown in Figure 2.

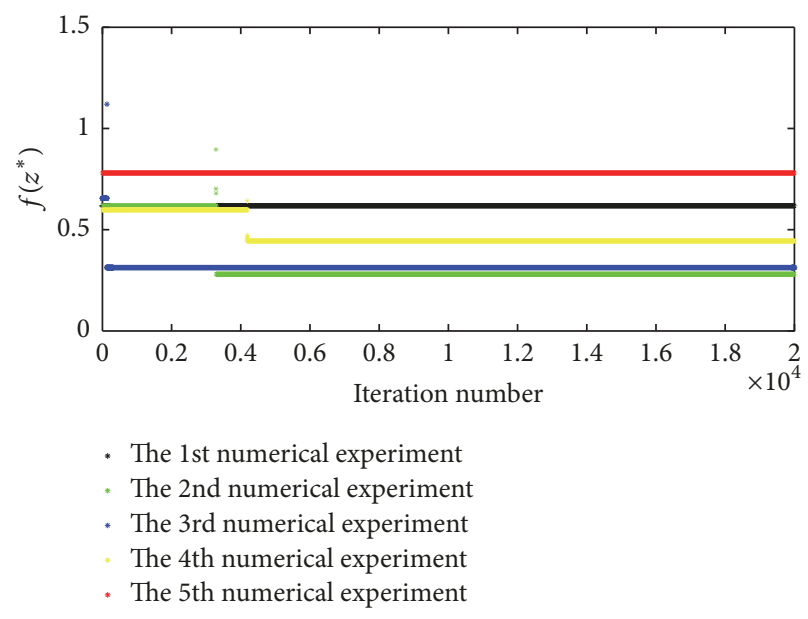

FIGURE 2: Change curve of numerical value of $f(z)$ at every iteration.

TABLE 2: Numerical results of Example 8.

\begin{tabular}{lcc}
\hline$\lambda$ & $x^{*}$ & $f\left(z^{*}\right)$ \\
\hline 0.8996 & $(0.4856,0.5973,0.3077,0.4007,0.5974,0.5284)^{T}$ & 0.5782 \\
0.9013 & $(0.6375,0.2817,0.3071,0.5837,0.3521,0.5110)^{T}$ & 0.2802 \\
1.0000 & $(0.2128,0.2264,0.0828,0.2039,0.1965,0.0488)^{T}$ & 0.3125 \\
0.9001 & $(0.5624,0.6134,0.4119,0.5258,0.5934,0.5930)^{T}$ & 0.4441 \\
1.0000 & $(0.0096,0.0049,0.0080,0.0014,0.0042,0.0091)^{T}$ & 0.7799 \\
\hline
\end{tabular}

Example 9. We consider problem (1), where

$$
\begin{aligned}
A(\omega) & =\left(\begin{array}{cccc}
2+\omega & 1 & \cdots & 1 \\
1 & 2+\omega & \cdots & 1 \\
\vdots & \vdots & \ddots & \vdots \\
1 & 1 & \cdots & 2+\omega
\end{array}\right)_{n \times n}, \\
B(\omega) & =\left(\begin{array}{cccc}
3+\omega & 1 & \cdots & 1 \\
1 & 3+\omega & \cdots & 1 \\
\vdots & \vdots & \ddots & \vdots \\
1 & 1 & \cdots & 3+\omega
\end{array}\right)_{n \times n}, \\
\Omega & =\{0,1\}, \\
p_{i} & =P\left(\omega_{i} \in \Omega\right)=0.5, \quad i=1,2 .
\end{aligned}
$$

When $n$ takes different values, the numerical results of Example 9 are shown in Table 3 and the values of $f(z)$ at each iteration are shown in Figure 3.

In the following, we consider the application of problem (1) in resonant cavity.

The calculation of the resonator can be described by the field equation and the boundary condition. With passivity 

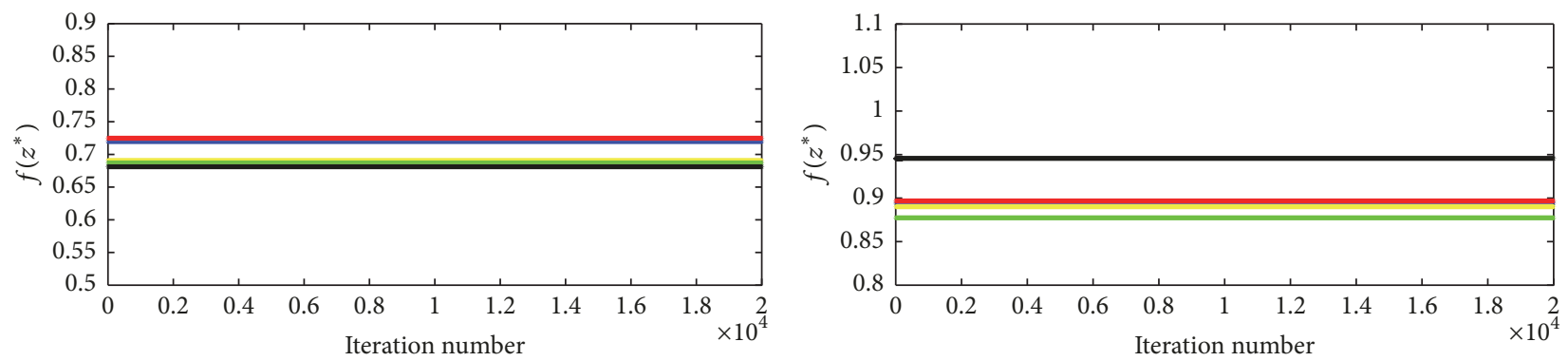

- The 1st numerical experiment

- The 2nd numerical experiment

- The 1st numerical experiment

- The 2nd numerical experiment

The 3rd numerical experiment

- The 4th numerical experiment

- The 3rd numerical experiment The 5th numerical experiment

* The 5th numerical experiment

(b) $\mathrm{DIM}=100$

(a) $\mathrm{DIM}=50$

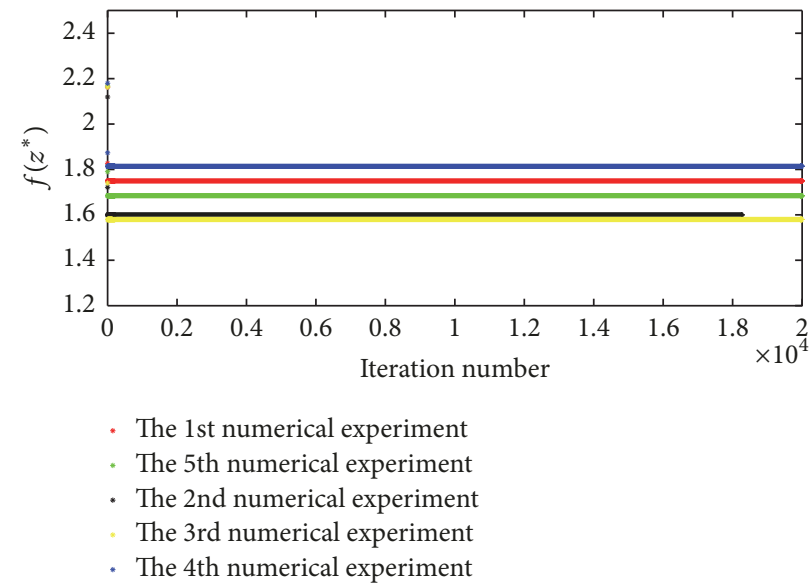

(c) $\mathrm{DIM}=200$

FIgURE 3: Change curve of numerical value of $f(z)$ at every iteration, when DIM takes different values.

TABLE 3: Numerical results of Example 9.

\begin{tabular}{lcc}
\hline DIM & $\lambda$ & $f\left(z^{*}\right)$ \\
\hline 50 & 1.4000 & 0.7196 \\
50 & 1.4000 & 0.7248 \\
50 & 1.4000 & 0.6907 \\
50 & 1.4000 & 0.6868 \\
50 & 1.4000 & 0.6811 \\
100 & 1.4000 & 0.9427 \\
100 & 1.4000 & 0.8941 \\
100 & 1.4000 & 0.8774 \\
100 & 1.4000 & 0.8967 \\
100 & 1.4000 & 0.8902 \\
200 & 1.6595 & 1.7401 \\
200 & 1.6610 & 1.6844 \\
200 & 1.6626 & 1.5796 \\
200 & 1.6584 & 1.8135 \\
200 & 1.6611 & 1.7051 \\
\hline
\end{tabular}

in the cavity, the field vector satisfies the homogeneous Helmholtz equation. The vector potential $A$ and the vector potential $A_{m}$ are used, and an arbitrary field vector $a$ is taken as a leading vector. We have

$$
\begin{aligned}
A & =a \Psi, \\
A_{m} & =a \Psi^{*},
\end{aligned}
$$

where $\Psi$ and $\Psi^{*}$ satisfy

$$
\begin{aligned}
\nabla^{2} \Psi+k^{2} \Psi & =0, \\
\nabla^{2} \Psi^{*}+k^{2} \Psi^{*} & =0 .
\end{aligned}
$$

On the inner wall $S$ of the cavity, the boundary conditions can often be converted to

$$
\begin{gathered}
\left.\Psi\right|_{S}=0, \\
\left.\Psi^{*}\right|_{S}=0 .
\end{gathered}
$$

Besides, as we all know, generally speaking, microwave engineering can be divided into two categories: eigenvalue problems and noneigenvalue problems. The problems of the propagation of electromagnetic wave in the waveguide and 
TABLE 4: Numerical results of Example 10.

\begin{tabular}{lcc}
\hline$\lambda$ & $x^{*}$ & $f\left(z^{*}\right)$ \\
\hline 1.1038 & $(0.0027,0.0073,0.0074,0.0034,0.0050)^{T}$ & 0.5255 \\
1.1097 & $(0.0010,0.0004,0.0055,0.0072,0.0075)^{T}$ & 0.5169 \\
1.1004 & $(0.0013,0.0057,0.0047,0.0001,0.0033)^{T}$ & 0.5172 \\
1.1718 & $(0.0065,0.0319,0.0129,0.0190,0.0034)^{T}$ & 0.6617 \\
1.1417 & $(0.0321,0.0181,0.0307,0.0310,0.0236)^{T}$ & 0.9004 \\
\hline
\end{tabular}

the distribution of the midfield in the cavity are all eigenvalue problems. In [38], the eigenvalue problem here is as follows:

$$
A x=\lambda x .
$$

The reason for such a conclusion is that if the problem is abstracted, the Laplace operator $\nabla^{2}$ acts on the function $\Psi$ and the product of matrix $A$ and vector $x$ can be regarded as a linear operator $L$ acting on an object $f$. follows:

In [3], the author considers the eigenvalue problem as

$$
K \varphi-\lambda B \varphi=0,
$$

where $K$ and $B$ are matrices, $\varphi$ is the unknown quantity, and $\lambda$ is the eigenvalue.

In a random system, different loop lengths can produce different resonant frequencies, resulting in multiple modes. So we denote the resonant frequencies as $\lambda \omega$, where $\omega$ is random.

In Example 10 of this paper, we transform the above eigenvalue problem into the eigenvalue complementarity problem (1) and consider the form in the stochastic system. Next, we give Example 10.

Example 10. We consider problem (1), where

$$
\begin{aligned}
B(\omega) & =\left(\begin{array}{ccccc}
12 \omega & \omega & -\omega & 2 \omega & \omega \\
\omega & 14 \omega & \omega & -\omega & \omega \\
-\omega & \omega & 16 \omega & -\omega & \omega \\
2 \omega & -\omega & -\omega & 12 \omega & -\omega \\
\omega & \omega & \omega & -\omega & 11 \omega
\end{array}\right), \\
K & =\left(\begin{array}{ccccc}
10 & 2 & 3 & 1 & 1 \\
2 & 12 & 1 & 2 & 1 \\
3 & 1 & 11 & 1 & -1 \\
1 & 2 & 1 & 9 & 1 \\
1 & 1 & -1 & 1 & 15
\end{array}\right), \\
\Omega & =\{0,1\}, \\
p_{i} & =P\left(\omega_{i} \in \Omega\right)=0.5, \quad i=1,2 .
\end{aligned}
$$

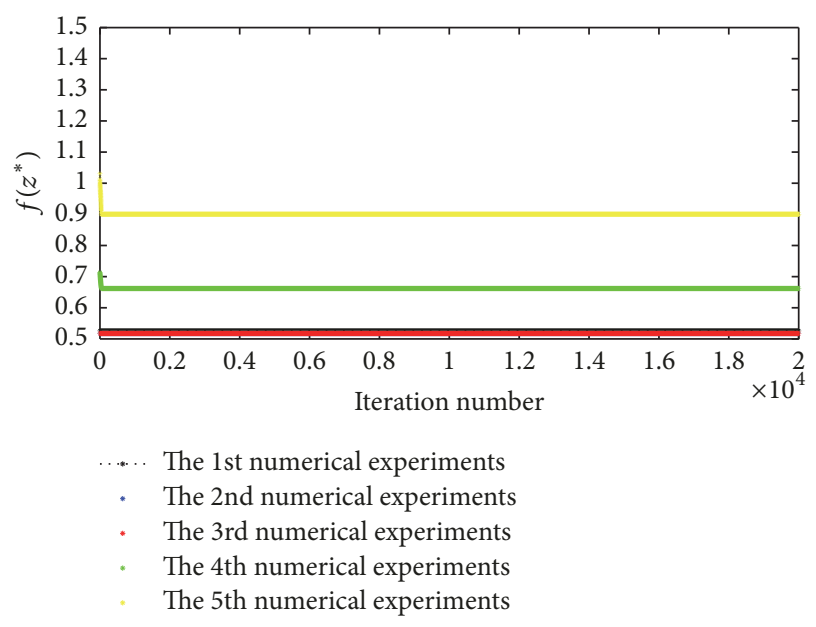

FIGURE 4: Change curve of numerical value of $f(z)$ at every iteration.

The numerical results of Example 10 are shown in Table 4 and the values of $f(z)$ at each iteration are shown in Figure 4.

\section{Conclusion}

The numerical experiments show that the projected smoothing Newton method is effective in solving the eigenvalue complementarity problems, even for high dimensional problems. After several experiments, we find that the value of $\mu$ in smoothing function has a great influence on the numerical results. When $\mu=0.01$, the numerical results are optimal. Using the smoothing Newton method, the objective function is polished. So the objective function is guided everywhere. Besides, the projected smoothing Newton method is Qquadratic. In the application of the projected smoothing Newton method, we consider the two-dimension resonator problem. Therefore, using the new technology of optimization method to further improve the accuracy and efficiency of the given method, the applications in more complicated resonator problems are of great significance and practical value.

\section{Conflicts of Interest}

The authors declare that there are no conflicts of interest regarding the publication of this paper.

\section{Acknowledgments}

This work was supported by National Natural Science Foundation of China (no. 11671220) and Natural Science Foundation of Shandong Province (no. ZR2016AM29).

\section{References}

[1] A. Taflove and S. C. Hagness, Computational Electrodynamics: The Finite-Difference Timedomain Method, Artech House, 2000.

[2] T. Maceina, P. Bettini, G. Manduchi, and M. Passarotto, "Fast and Efficient algorithms for computational electromagnetics on 
GPU architecture," IEEE Transactions on Nuclear Science, vol. 64, no. 7, pp. 1983-1987, 2017.

[3] C. Q. Wang, The Basis of Modern Computational Electromagnetics, Peking University press, 2005.

[4] S. M. Anlage, J. Rodgers, S. Hemmady, J. Hart, T. M. Antonsen, and E. Ott, "New results in chaotic time-reversed electromagnetics: high frequency one-recording-channel time-reversal mirror," Acta Physica Polonica A, vol. 112, no. 4, pp. 569-574, 2007.

[5] X. Gao, C. Sui, S. Hemmady et al., "Predicting EMI induced delay errors in integrated circuits: sensitivity to the velocity saturation index," Electromagnetic Compatibility, pp. 102-105, 2015.

[6] S. Clénet, "Uncertainty quantification in computational electromagnetics: the stochastic approach," International Compumag Society Newsletters, 2013.

[7] Y. Tawk, M. Al-Husseini, S. Hemmady, and A. R. Albrecht, "Statistical prediction and measurement of induced voltages on components within complicated enclosures: a wave-chaotic approach," in International Conference on Electromagnetics in Advanced Applications, vol. 17, pp. 294-297, 2010.

[8] J. J. Júdice, H. D. Sherali, and I. M. Ribeiro, "The eigenvalue complementarity problem," Computational optimization and applications, vol. 37, no. 2, pp. 139-156, 2007.

[9] J. Júdice, M. Raydan, S. S. Rosa, and S. A. Santos, "On the solution of the symmetric eigenvalue complementarity problem by the spectral projected gradient algorithm," Numerical Algorithms, vol. 47, no. 4, pp. 391-407, 2008.

[10] L. S. Fernandes, J. J. Júdice, M. Fukushima, and A. Iusem, "On the symmetric quadratic eigenvalue complementarity problem," Optimization Methods \& Software, vol. 29, no. 4, pp. 751-770, 2014.

[11] H. A. Le Thi, M. Moeini, T. Pham Dinh, and J. Judice, "A DC programming approach for solving the symmetric eigenvalue complementarity problem," Computational Optimization and Applications, vol. 51, no. 3, pp. 1097-1117, 2012.

[12] Y.-Y. Chen and Y. Gao, "Levenberg-marquardt method for the eigenvalue complementarity problem," The Scientific World Journal, vol. 2014, Article ID 307823, 6 pages, 2014.

[13] J. J. Júdice, H. D. Sherali, I. . Ribeiro, and S. S. Rosa, "On the asymmetric eigenvalue complementarity problem," Optimization Methods \& Software, vol. 24, no. 4-5, pp. 549-568, 2009.

[14] A. Pinto da Costa, J. A. Martins, I. N. Figueiredo, and J. J. Júdice, "The directional instability problem in systems with frictional contacts," Computer Methods Applied Mechanics and Engineering, vol. 193, no. 3-5, pp. 357-384, 2004.

[15] X. Chen and M. Fukushima, "Expected residual minimization method for stochastic linear complementarity problems," Mathematics of Operations Research, vol. 30, no. 4, pp. 1022-1038, 2005.

[16] G. L. Zhou and L. Caccetta, "Feasible semismooth Newton method for a class of stochastic linear complementarity problems," Journal of Optimization Theory and Applications, vol. 139, no. 2, pp. 379-392, 2008.

[17] X. Chen, C. Zhang, and M. Fukushima, "Robust solution of monotone stochastic linear complementarity problems," Mathematical Programming, vol. 117, no. 1-2, pp. 51-80, 2009.

[18] C. Ling, L. Qi, G. Zhou, and L. Caccetta, "The $S C^{1}$ property of an expected residual function arising from stochastic complementarity problems," Operations Research Letters, vol. 36, no. 4, pp. 456-460, 2008.
[19] Y. W. Huang and G. H. Lin, "Some results on solving a class of stochastic mathematical programs with complementarity constraints," Operations Research and Fuzziology, vol. 02, no. 03, pp. 35-41, 2012.

[20] Z. Liu, S. Du, and R. Wang, "A new conjugate gradient projection method for solving stochastic generalized linear complementarity problems," Journal of Applied Mathematics and Physics, vol. 04, no. 06, pp. 1024-1031, 2016.

[21] H. Fang, X. Chen, and M. Fukushima, "Stochastic $R_{0}$ matrix linear complementarity problems," SIAM Journal on Optimization, vol. 18, no. 2, pp. 482-506, 2007.

[22] G.-H. Lin and M. Fukushima, "Stochastic equilibrium problems and stochastic mathematical programs with equilibrium constraints: a survey," Pacific Journal of Optimization. An International Journal, vol. 6, no. 3, pp. 455-482, 2010.

[23] K. Hamatani and M. Fukushima, "Pricing american options with uncertain volatility through stochastic linear complementarity models," Computational Optimization and Applications, vol. 50, no. 2, pp. 263-286, 2011.

[24] B. Chen and P. T. Harker, "Smooth approximations to nonlinear complementarity problems," SIAM Journal on Optimization, vol. 7, no. 2, pp. 403-420, 1997.

[25] C. H. Chen and O. L. Mangasarian, "Smoothing methods for convex inequalities and linear complementarity problems," Mathematical Programming, vol. 71, no. 1, pp. 51-69, 1995.

[26] C. Chen and O. L. Mangasarian, "A class of smoothing functions for nonlinear and mixed complementarity problems," Computational Optimization and Applications, vol. 5, no. 2, pp. 97-138, 1996.

[27] B. Chen, X. Chen, and C. Kanzow, "A penalized FischerBurmeister NCP-function," Mathematical Programming, vol. 88, pp. 211-216, 2000.

[28] J. Y. Han, N. H. Xiu, and H. D. Qi, Nonlinear Complementary Theory and Algorithm, Shanghai Scientific and Technical Publishers, Shanghai, China, 2006.

[29] Y. K. Huang, The Research of the Algorithms of the Stochastic Linear Complementarity Problems, Xidian University, Xi'an, China, 2010.

[30] C. Kanzow and H. Pieper, "Jacobian smoothing methods for nonlinear complementarity problems," SIAM Journal on Optimization, vol. 9, no. 2, pp. 342-373, 1999.

[31] L. Qi, D. Sun, and G. Zhou, "A new look at smoothing Newton methods for nonlinear complementarity problems and box constrained variational inequalities," Mathematical Programming, vol. 87, no. 1, pp. 1-35, 2000.

[32] X. Chen, L. Qi, and D. Sun, "Global and superlinear convergence of the smoothing Newton method and its application to general box constrained variational inequalities," Mathematics of Computation, vol. 67, no. 222, pp. 519-540, 1998.

[33] X. Chen, "Smoothing methods for nonsmooth, nonconvex minimization," Mathematical Programming, vol. 134, no. 1, pp. 71-99, 2012.

[34] L. Q. Qi and X. J. Chen, "A globally convergent successive approximation method for severely nonsmooth equations," SIAM Journal on Control and Optimization, vol. 33, no. 2, pp. 402-418, 1995.

[35] L. Q. Qi, "Convergence analysis of some algorithms for solving nonsmooth equations," Mathematics of Operations Research, vol. 18, no. 1, pp. 227-244, 1993.

[36] F. H. Clarke, Optimization and Nonsmooth Analysis, John Wiley and Sons, New York, NY, USA, 1983. 
[37] C. Kanzow and S. Petra, "Projected filter trust region methods for a semismooth least squares formulation of mixed complementarity problems," Optimization Methods \& Software, vol. 22, no. 5, pp. 713-735, 2007.

[38] F. Xing, Numerical Calculation and Simulation Analysis of Electromagnetic Field, National Defense Industry Press, 2014. 


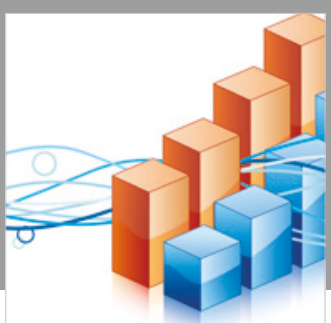

Advances in

Operations Research

\section{-n-m}
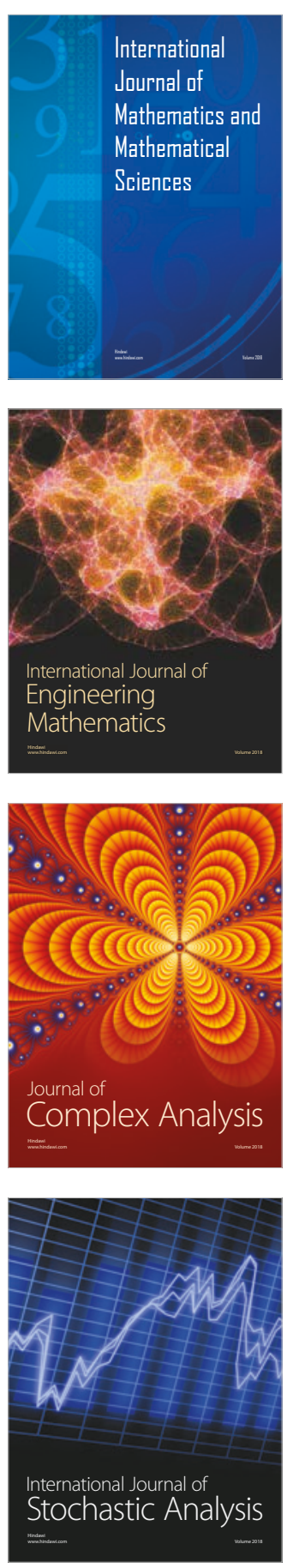
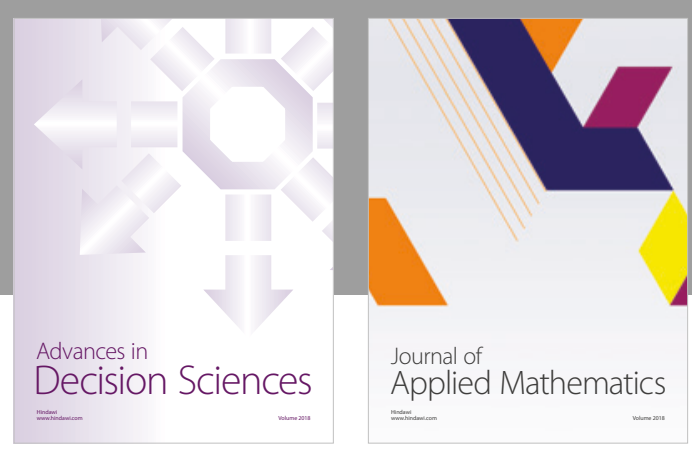

Journal of

Applied Mathematics
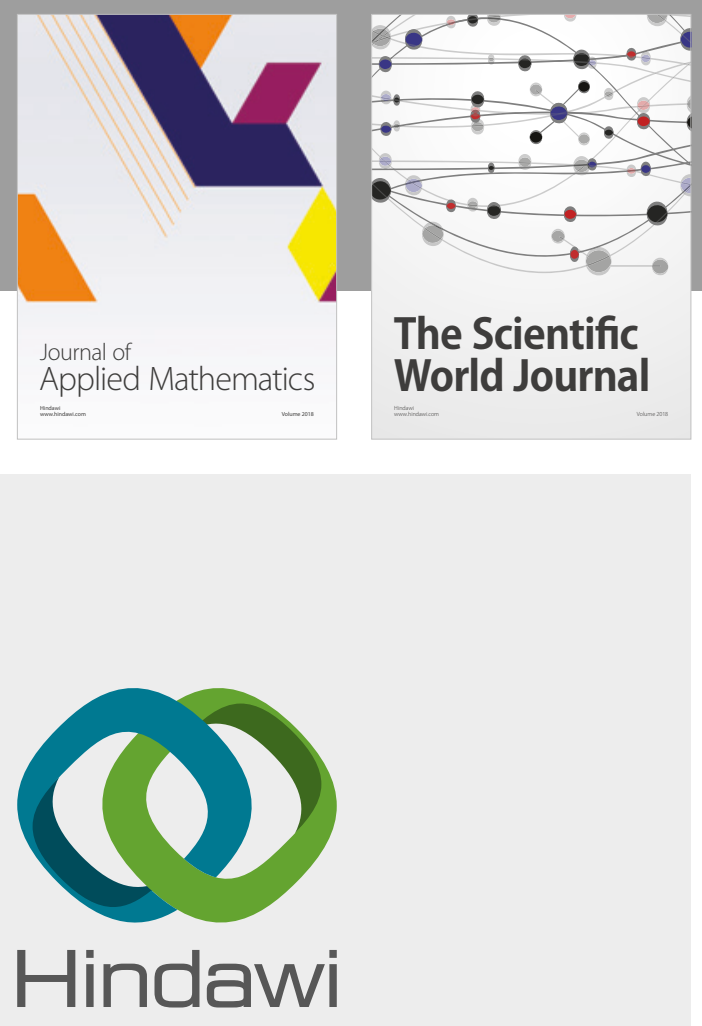

Submit your manuscripts at

www.hindawi.com

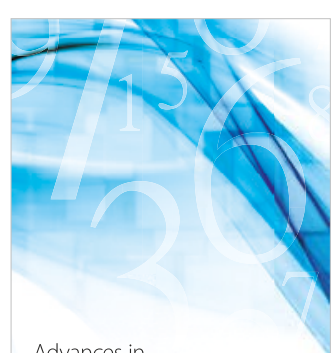

Advances in
Numerical Analysis
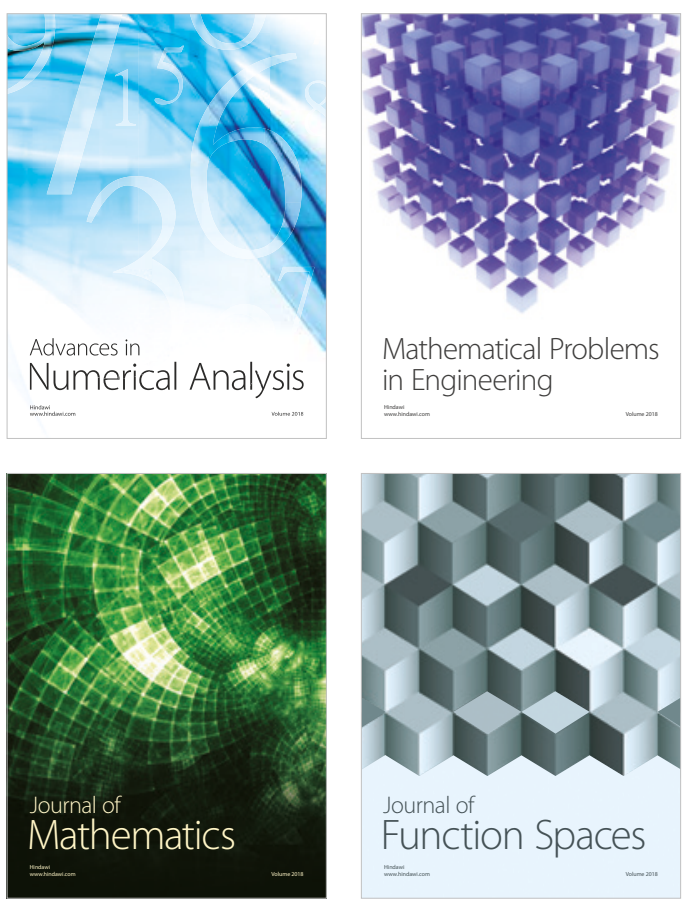

Mathematical Problems in Engineering

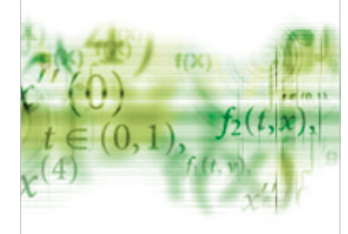

International Journal of

Differential Equations

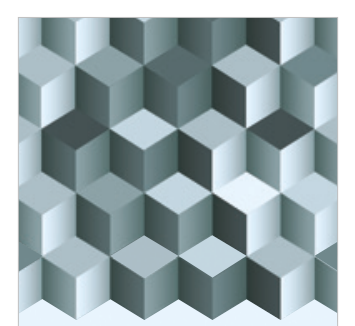

Journal of

Function Spaces

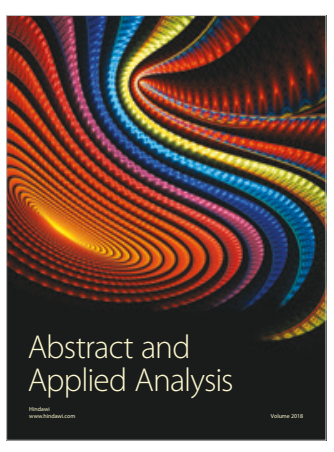

The Scientific

World Journal

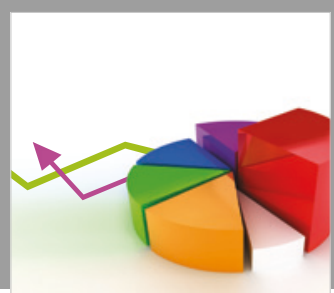

Journal of

Probability and Statistics
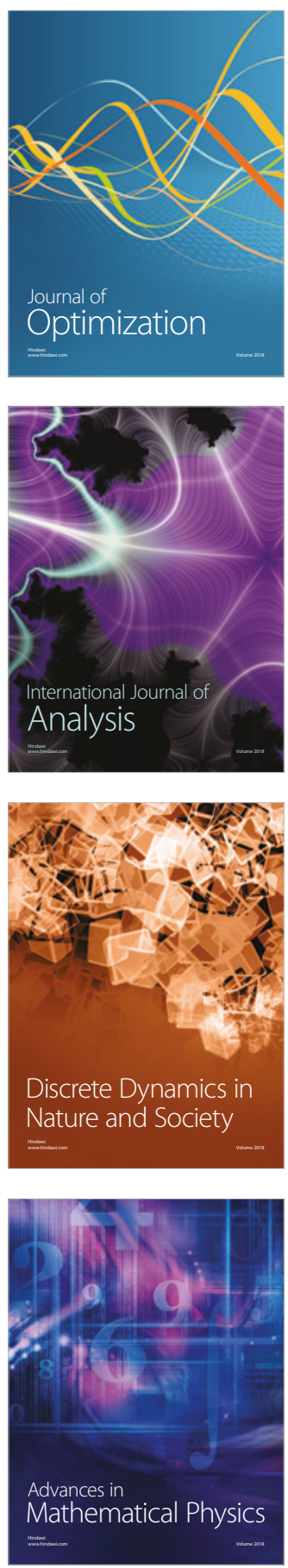